\title{
Bond compressibility and bond Grüneisen parameters of CdTe
}

\author{
P. Fornasini ${ }^{1}$, R. Grisenti ${ }^{1}$, T. Irifune ${ }^{2}$, T. Shinmei ${ }^{2}$, O.

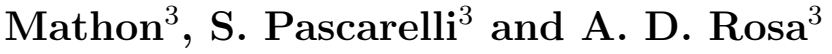 \\ ${ }^{1}$ Dipartimento di Fisica - Università di Trento, Via Sommarive 14, I-38123 Povo \\ (Trento), Italy. \\ ${ }^{2}$ Geodynamics Research Center, Ehime University, Matsuyama, Japan \\ ${ }^{3}$ ESRF, Grenoble, France \\ E-mail: paolo.fornasini@unitn.it
}

\begin{abstract}
Extended X-ray absorption fine structure (EXAFS) at the Cd K edge and diffraction patterns have been measured on $\mathrm{CdTe}$ as a function of pressure from $100 \mathrm{kPa}$ (1 bar) to $5 \mathrm{GPa}$ using a cell with nano-polycrystalline diamond anvils and an X-ray focussing scanning spectrometer. Three phases - zincblende (ZB), mixed cinnabar-ZB and rocksalt (RS) - are well distinguished in different pressure intervals. The bond compressibility measured by EXAFS in the ZB phase is slightly smaller than the one measured by diffraction and decreases significantly faster when the pressure increases; the difference is attributed to the effect of relative vibrations perpendicular to the Cd-Te bond. The parallel mean square relative displacement (MSRD) decreases, the perpendicular MSRD increases when the pressure increases, leading to an increasing anisotropy of relative atomic vibrations. A constant-temperature bond Grüneisen parameter (GP) has been evaluated for the ZB phase and compared with the constantpressure bond GP measured in a previous experiment; an attempt is made to connect the bond GPs measured by EXAFS and the more familiar thermodynamic GP and mode GPs; the comparisons suggest the inadequacy of the quasi-harmonic approximation to deal with the local vibrational properties sampled by EXAFS.
\end{abstract}

PACS numbers: 07.35.+k,61.05.cj,62.50.-p,65.40.G-

Submitted to: J. Phys.: Condens. Matter 


\section{Introduction}

It is well known that a number of local structural and vibrational properties of crystalline solids cannot be directly inferred from the knowledge of the corresponding average properties. For that reason, extended X-ray absorption fine structure (EXAFS), which directly probes local properties, is considered a complementary technique to diffraction (Bragg scattering). As a matter of fact, one of the first and most striking successes of EXAFS has been the detection of the local distortions in crystalline random ternary alloys [1]. Non-negligible differences between average and local properties are present even in simple ordered crystals; a well known example is the large difference between the EXAFS and diffraction Debye-Waller factors, due to the effect of correlation [2]; less dramatic but often significant is the difference of the interatomic distances measured by EXAFS and diffraction, which is due to the effect of perpendicular vibrations $[3,4,5,6]$.

The differences between local and average properties in simple crystals have been thoroughly studied by temperature-dependent measurements. Recent results for a number of different crystalline systems include the evaluation of: a) the temperature dependence of the coefficient of bond thermal expansion [7], which is different from the coefficient of lattice expansion, $b$ ) the three-dimensional distribution of vector distances $[8,9]$, which is anisotropic also when the atomic thermal ellipsoids are isotropic, $c$ ) the anharmonic contributions to the second cumulant (Debye-Waller exponent) of the first coordination shell in CdTe [10].

In the last case, an attempt has also been made to define a constant-pressure bond Grüneisen parameter (GP), calculated as the logarithmic derivative, with respect to the bond distance, of the temperature-dependent Einstein frequency best fitting the second cumulant [10]. No clear relationship was found between the so-obtained constant-pressure bond GP and the more familiar thermodynamic GP [11] or the constant-temperature mode GPs [12], which are defined within the framework of the quasi-harmonic approximation (QHA). The inadequacy of the QHA to deal with the vibrational properties of the negative-thermal-expansion crystal $\mathrm{ScF}_{3}$ has been recently claimed by several authors $[13,14]$; these findings open a more general question about the suitability of the quasi-harmonic approximation to explain the local vibrational properties probed by EXAFS.

EXAFS measurements as a function of pressure have been performed since long time on a number of systems $[15,16,17,18,19,20]$. Less attention has been paid to the differences between local and average properties of crystals in pressure-dependent $[21,22,23,24,25]$ than in temperature-dependent measurements. Concerning relatively simple systems, a slightly lower compressibility was found by San-Miguel et al. for ZnTe from EXAFS with respect to diffraction [18]. The pressure effects on the EXAFS DebyeWaller factors of $\mathrm{Cu}, \mathrm{Kr}, \mathrm{Si}, \mathrm{Ge}$ and $\mathrm{Pt}$ have been calculated by anharmonic Einstein or correlated Debye models [26, 27, 28].

Recently, the development of nano-polycrystalline diamond (NPD) anvil cells $[29,30,31]$, which eliminate the glitches due to Bragg scattering from EXAFS spectra, 
has greatly contributed to increase the measurements accuracy [32, 33, 34]. A further increase of accuracy is guaranteed by the possibility of performing measurements by an energy scanning spectrometer equipped with an X-ray focussing device [35, 36], which allows one to obtain a longer $k$ range and a higher $\mathrm{S} / \mathrm{N}$ ratio with respect to dispersion devices. An example is the recent study of the high-pressure phase VI of metallic Rb [37], where an attempt was also made at estimating the constant-temperature zero-pressure bond GP; only semi-quantitative considerations could be made, owing to the complex structure of the crystal, characterized by a rich distribution of distances; moreover, the connection between bond GP and thermodynamic GP could not be clarified.

In the light of the achievements in temperature-dependent EXAFS measurements, it appears appealing to take advantage of the recent developments in instrumentation for pressure-dependent experiments, such as NPD anvil cells and X-ray focussing scanning spectrometers, to verify the possible differences between bond and lattice compressibilities as well as to evaluate the constant-temperature bond GPs. These findings are expected to give a deeper understanding of the local thermodynamic behaviour of crystals, and in particular of the relationships between the differently defined GPs and of the validity of the QHA.

To that aim, we choose to measure EXAFS spectra and diffraction patterns on the II-VI compound CdTe in the pressure range from $100 \mathrm{kPa}$ (1 bar) to $5 \mathrm{GPa}$. The choice of CdTe was suggested by the availability of high quality temperature-dependent data previously measured at both $\mathrm{Cd}$ and Te edges [38, 39], which facilitates the comparison between temperature-dependent and pressure-dependent measurements, and by the relatively high degree of anharmonicity, which enhances the difference between local and average behaviour and should enlighten the possible failure of the QHA.

Much attention has already been devoted to the pressure-dependent behaviour of II-VI compounds [40]. Diffraction measurements on CdTe have been performed at room temperature by Nelmes et al. up to $28 \mathrm{GPa}[41,42,43]$. When increasing pressure, the zincblende $(\mathrm{ZB})$ phase (space group $F \overline{4} 3 \mathrm{~m}$ ) is stable up to about $3.53 \mathrm{GPa}$, where an intermediate cinnabar-like phase appears (space group $P 3{ }_{1} 21$ ) and progressively substitutes the ZB phase. At $3.8 \mathrm{GPa}$, a transition to a rocksalt (RS) phase occurs (space group $F m \overline{3} m$ ). At $10.1 \mathrm{GPa}$, CdTe undergoes a continuous transition from the $\mathrm{RS}$ to a site-ordered phase with $\mathrm{Cmcm}$ structure, which is stable at least up to $28 \mathrm{GPa}$ [43]. The phase transitions of CdTe under pressure have been reproduced by Côté et al through ab initio calculations based on a pseudopotential total-energy scheme [44].

The present work is chiefly focused on the ZB phase of CdTe and its main aims are: a) to compare the bond compressibility and the lattice compressibility measured by EXAFS and by diffraction, respectively; $b$ ) to measure the pressure dependence of the second and third EXAFS cumulants and to separate the harmonic from the anharmonic contributions to the second cumulant; c) to calculate a constant-temperature bond GP from the logarithmic derivative of the Einstein frequency with respect to the bond length and to compare it with the previously measured bond GP at constant pressure [10] and with the mode GPs of the QHA; d) to evaluate the three-dimensional distribution of 
vector distances and monitor the evolution of its anisotropy when the pressure increases.

The measurements have been extended to the mixed cinnabar-ZB and to the RS phases, mainly to check the consistency with previous diffraction studies. A particular attention has been devoted to the relatively high value of the Debye-Waller factor in the RS phase.

The paper is organized as follows. Sections 2 and 3 are dedicated to some details about the experiment and the data analysis procedures. The experimental results are presented in Section 4. In Section 5, the discussion is focussed on the difference between bond and lattice compressibilities, on the anisotropy of relative vibrations and on the definition and comparison of different Grüneisen parameters. Section 6 is dedicated to conclusions.

\section{Experimental details}

The sample was prepared from the same CdTe powder $99.999 \%$ pure (from Alfa Aesar) previously used for temperature-dependent measurements [38]. Transmission EXAFS and diffraction measurements as a function of pressure were performed at the BM23 beamline [35] of the European Synchrotron Radiation Facility (ESRF) in Grenoble, France. Electron energy and average current were $6 \mathrm{GeV}$ and $190 \mathrm{~mA}$, respectively.

The sample was loaded, together with silicone oil as a pressure medium and a ruby crystal serving as pressure standard, in a LeToullec-type membrane diamond anvil cell [45]. Silicone oil acts as a quasi hydrostatic pressure transmission medium up to 3 GPa, while it induces minor pressure gradients of less than $0.25 \mathrm{GPa}$ at $5 \mathrm{GPa}$ [46]. Novel sintered NPD anvils [29] were employed with a culet size of 600 microns, which allow to acquire glitch-free EXAFS spectra in any energy range [30, 37]. The sample was contained in a steel gasket, pre-indented to 80 micron thickness having a laser-drilled sample cavity of 250 micron in diameter in the center.

The X-ray beam was tuned to the energy of the Cd K-edge $(26.711 \mathrm{keV})$ by a doublecrystal fixed-exit monochromator equipped with two $\mathrm{Si}(311)$ crystals, whose relative energy resolution was $1.9 \times 10^{-5}$. Beam focusing to $3 \times 3 \mu \mathrm{m}^{2}$ and harmonic rejection was achieved through a Kirkpatrick-Baez mirror system with Pt coating inclined to a rejection angle of $2.5 \mathrm{mrad}[36,37]$. The monochromator angle was calibrated using a Cd-foil prior and several times during the experiment.

Angle-dispersive diffraction images were collected at an X-ray energy of $26.0 \mathrm{keV}$, using a Mar165 CCD detector placed $237 \mathrm{~mm}$ from the sample. The sample-to-detector distance, the beam center position and the detector tilt angles were determined using a $\mathrm{CeO}_{2}$ standard and the program DIOPTAS [47].

For XAS measurements at the $\mathrm{Cd} \mathrm{K}$ edge, the energy of the incident X-rays was scanned in the range $E=26.4-28.2 \mathrm{keV}$, with a $\Delta E$ step varying from $0.5 \mathrm{eV}$ in the near-edge region to $5 \mathrm{eV}$ at the end of the spectra, in order to obtain a uniform wavevector step $\Delta k=0.025 \AA$ in the EXAFS region. The incoming and outgoing photon fluxes were measured by two $50 \mathrm{~mm}$ long ionization chambers operating at $1000 \mathrm{~V}$ and 
filled with krypton gas, at pressures of 0.78 and 2.58 bar, respectively. The measured edge jump $\Delta \mu x$ at the $\mathrm{Cd} \mathrm{K}$ edge was about 0.7 .

The sample pressure was varied in steps of $0.2-0.3 \mathrm{GPa}$ starting from $100 \mathrm{kPa}$ up to $5 \mathrm{GPa}$. The pressure was evaluated by measuring the shift of the fluorescent $R_{2}$ line of ruby; the uncertainty of pressure values was assumed $\pm 0.03 \mathrm{GPa}[46,40]$. At each pressure step, after stabilization of the pressure, both EXAFS and diffraction data were acquired, with an exposure time of $5 \mathrm{~min}$ for the diffraction images and 1-2 hours for the collection of typically three EXAFS spectra. Because of the long acquisition time, the pressure was measured before and after diffraction and EXAFS measurements at each pressure point, to account for pressure variations. At the end of the experiment, the pressure was lowered to the ambient value $100 \mathrm{kPa}$ and eight EXAFS spectra were measured on the quenched sample, to serve as reference for the subsequent analysis

\section{Data analysis}

The extraction of the EXAFS functions $\chi(k)$ from the experimental spectra was performed according to a well established procedure [48, 39]. The values of the photoelectron wave-vector $k$ were calculated with respect to an edge energy conventionally set at the maximum of the first derivative of one of the $100 \mathrm{kPa}$ spectra (measured at the end of the experiment), chosen as reference. The energy axes of the other spectra measured at all pressures were then shifted to achieve the best superposition with the reference spectrum in the edge region and the origin of the $k$ axis of each spectrum was determined accordingly.

EXAFS functions at three different pressures, corresponding to three different phases, are shown in figure 1. Thanks to the direct transmission measurements and the use of NPD anvils, high-quality glitch-free signals have been obtained up to at least $16 \AA^{-1}$. The $100 \mathrm{kPa}$ EXAFS function (a) is the average of the EXAFS functions of the eight spectra measured at the end of the experiment. All EXAFS functions are characterized by a single dominant frequency, indicating negligible contributions of the coordination shells beyond the first one.

The $k$-weighted functions $k^{2} \chi(k)$, multiplied by a Gaussian window, were Fourier transformed in the $k$ range from 1.9 to $16 \AA^{-1}$. The Fourier transforms (FT) at three different pressures, corresponding to the three different phases, are compared in figure 2. The contribution of the second and outer shells is negligible in all phases. For the ZB phase at $100 \mathrm{kPa}$, the $\mathrm{FT}$ is in good agreement with the one obtained at the same temperature and pressure in previous experiments performed as a function of temperature [38, 39]. The first-shell peak in the RS phase is significantly lower than in the ZB phase, in spite of the larger coordination number (6 against 4$)$, suggesting a large Debye-Waller factor.

To get quantitative results, the 1st-shell EXAFS functions were parametrized in terms of the leading cumulants of the distance distribution [49, 17]: the first cumulant is the average value $\langle r\rangle$, the second cumulant is the variance of the 


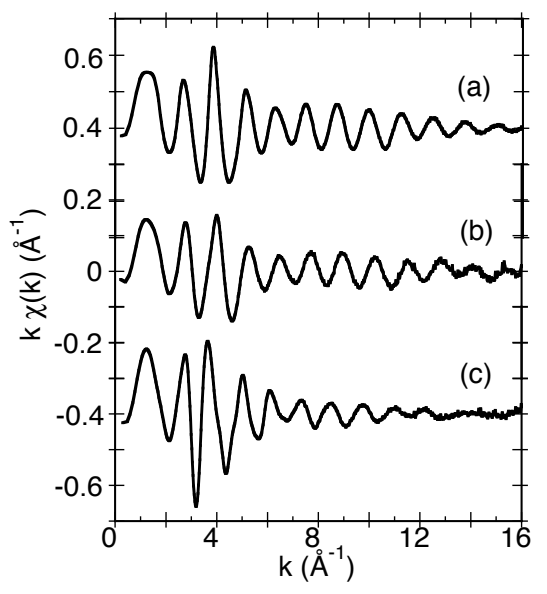

Figure 1. EXAFS functions $k \chi(k)$ at the $\mathrm{Cd} \mathrm{K}$ edge in $\mathrm{CdTe}$ at three different pressures: (a) $100 \mathrm{kPa}$ (ZB phase), (b) $3.79 \mathrm{GPa}$ (mixed $\mathrm{ZB}+$ cinnabar phase),(c) 4.75 GPa (RS phase).

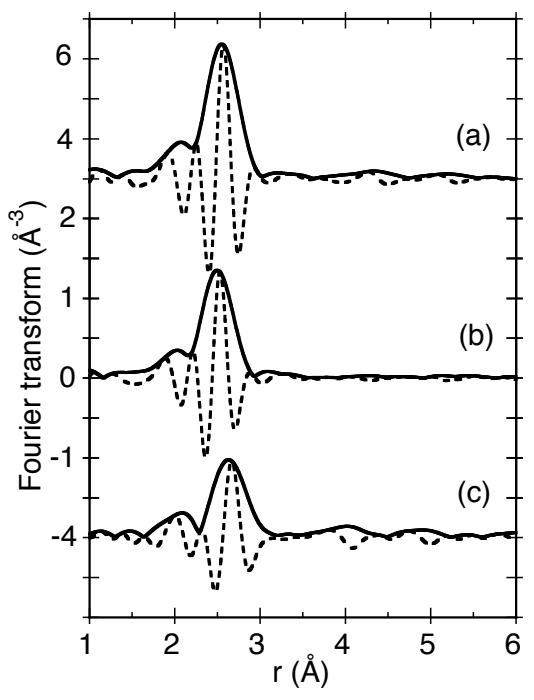

Figure 2. Fourier transforms of the functions $k^{2} \chi(k)$ at three different pressures: (a) $100 \mathrm{kPa}$ (ZB phase), (b) $3.79 \mathrm{GPa}$ (mixed ZB+cinnabar phase), (c) $4.75 \mathrm{GPa}$ (RS phase). Dashed and continuous lines are the imaginary part and the modulus, respectively.

distribution and corresponds to the parallel mean square relative displacement (MSRD) or EXAFS Debye-Waller exponent, the third cumulant depends on the asymmetry of the distribution [9]. Two different and somewhat complementary analysis procedures were carried out.

In the first procedure, the ratio method [49, 50, 51], the first-shell contribution to the EXAFS function of the different spectra was obtained by Fourier back-transforming the corresponding peaks in the $r$-space and the phases and amplitudes were separately analyzed, taking as reference the average of the eight spectra measured at $100 \mathrm{kPa}$ 
at the end of the experiment. The ratio method gives the relative values $\delta C_{n}$ of the leading cumulants of an effective distribution $P(r, \lambda)=\rho(r) e^{-2 r / \lambda} / r^{2}$, where $\rho(r)$ is the real distribution of distances and $\lambda$ is here the photoelectron mean free path. The cumulants of the real distribution, $C_{n}^{*}$, were derived from the cumulants of the effective distribution, $C_{n}$, according to the recursion formula of [48]; the electron mean free path was fixed to the value $\lambda=9 \pm 3 \AA$. The difference between the cumulants of the real and effective distributions was significant only for the first cumulant.

In the second procedure, backscattering amplitudes, phase-shifts and inelastic terms were calculated by using the FEFF6 code $[52,53]$ and a non-linear best-fit of theoretical to experimental spectra was performed by the IFEFFIT code [54] using the graphical interface Artemis [55]. A good agreement was found between the two procedures for what concerns the relative values of the cumulants in the ZB phase. The evaluation of the absolute values of the $\mathrm{Cd}$-Te bond distance is discussed in Sections 4.2 and 4.4 for the ZB and RS phases, respectively.

The uncertainty of the relative values of cumulants was evaluated according to the procedures detailed in [39].

Diffraction images were integrated using the software FIT2D [56]. The diffraction patterns were further processed by the Rietveld refinement method using the software package MAUD [57]. From the refinements, the crystallographic parameters and average volume proportions of the different phases of CdTe were extracted.

\section{Results}

\subsection{Overview of the different phases}

The three different phases (ZB, mixed ZB-cinnabar and RS) are well singled out in the diffraction patterns. Three examples, measured at 3.65, 3.8 and $4.75 \mathrm{GPa}$, respectively, are shown in figure 3 . The relatively large peak appearing at $2 \theta=13.3^{\circ}$ in all patterns is the (111) reflection from the anvil polycrystalline diamond. In the bottom panel, the peaks at $2 \theta=7.31,11.95,14.04^{\circ}$ correspond to the (111), (220) and (311) reflections of the ZB phase of $\mathrm{CdTe}$, respectively. In the middle panel, the peaks of the ZB phase are still present, although reduced in intensity with respect to the diamond peak and accompanied by a number of peaks of the cinnabar phase. In the top panel, the peak at $2 \theta=9.28^{\circ}$, the shoulder at $2 \theta=13.14^{\circ}$ and the peak at $2 \theta=16.11^{\circ}$ correspond to the (200), (220) and (222) reflections of the RS phase, respectively.

The structural evolution with pressure is monitored also by the modifications of the XANES region of the absorption spectra (figure 4). The XANES at the Cd K edge of CdTe and their modifications are much weaker than the ones observed at both the $\mathrm{Cu}$ and $\mathrm{Br}$ edges of $\mathrm{CuBr}$ [16] and at the Zn K edge of ZnTe [18], owing to the larger energy width of the excited state and to the corresponding poorer energy resolution. The XANES of CdTe are unchanged in the ZB phase up to $3.65 \mathrm{GPa}$. In the pressure range from 3.67 to $4.07 \mathrm{GPa}$, the $a, b$ and $c$ structures are progressively modified, as a 


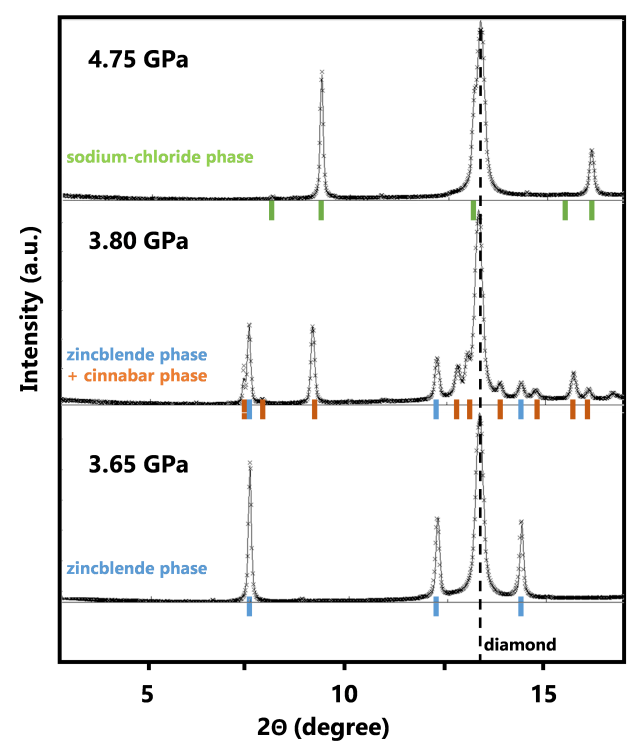

Figure 3. Diffraction patterns obtained by integrating the 2D detector signals over the $2 \pi$ azimuthal angle. Bottom: pure ZB phase measured at $3.65 \mathrm{GPa}$. Middle: mixed ZB-cinnabar phase measured at 3.8 GPa. Top: pure RS phase measured at $4.75 \mathrm{GPa}$.

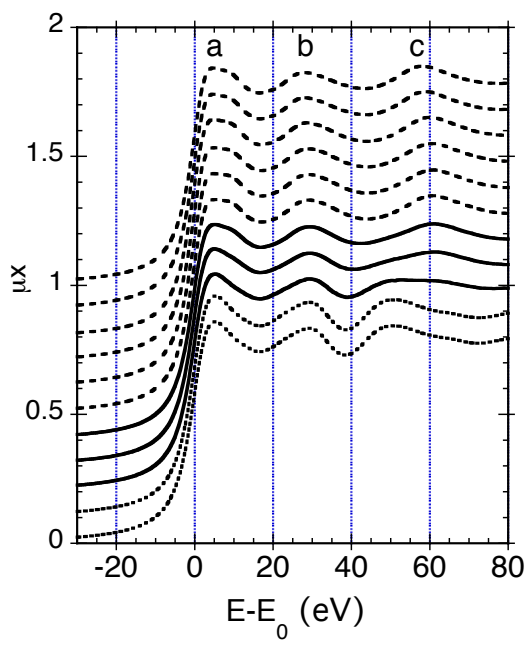

Figure 4. XANES structures at the $\mathrm{Cd} \mathrm{K}$ edge in CdTe at various pressures, increasing from top to bottom. Dashed lines: ZB phase, $100 \mathrm{kPa}$ and 1.07, 2.49, 3.0, 3.56, 3.65 GPa. Continuous lines: cinnabar+ZB, 3.67, 3.83, 4.07 GPa. Dotted lines: RS phase, 4.7 and $5.3 \mathrm{GPa}$. 


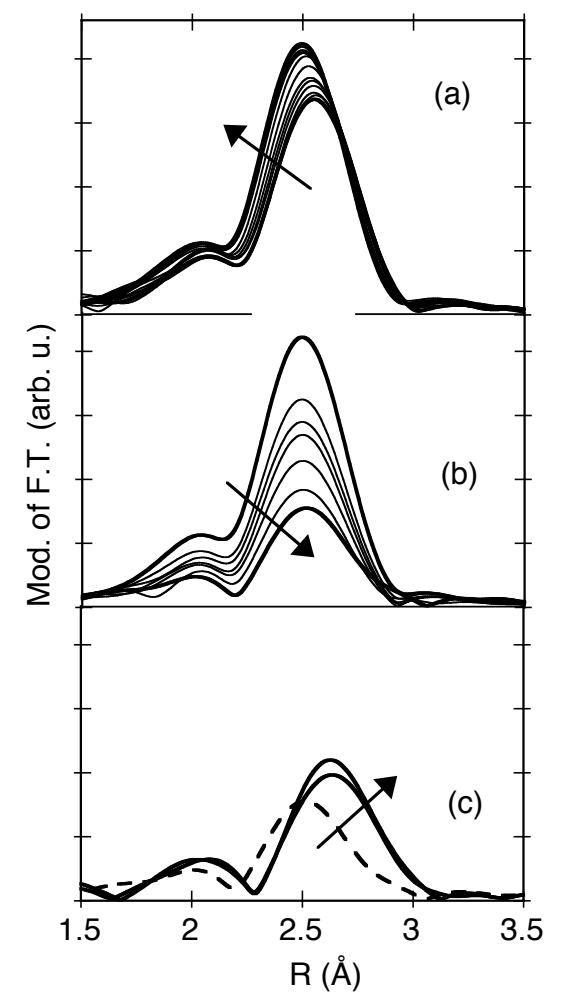

Figure 5. Moduli of Fourier Transforms in the first-shell region at different pressures. The arrows show the direction of increasing pressure. (a) ZB phase. (b) Mixed ZBcinnabar phases. (c) Highest-pressure cinnabar phase (dashed line) and RS phase (continuous lines).

signature of the progressive substitution of the ZB phase with the cinnabar phase. The transition to the RS phase is characterized by a relatively weak modification of the $a$ structure and a stronger modification of the $b$ and $c$ structures.

Much more evident than in the XANES are the pressure effects on the FTs of the EXAFS functions (figure 5). In the ZB phase (panel a) the increase of pressure is accompanied by an increase of the peak height and its shift towards smaller distances, corresponding to a decrease of the vibrational Debye-Waller factor and of the interatomic distance, respectively. The onset and the progressive development of the cinnabar phase (panel b) are accompanied by a strong progressive reduction of the first-shell peak, reasonably caused by the increase of the static Debye-Waller factor due to the presence of four different first-shell distances (one in the ZB phase and three in the cinnabar phase). The RS phase (panel c) is characterised by a quite small first-shell peak. Since the coordination number six in the RS phase is larger than the coordination number four in the ZB phase, the significant reduction of the first-shell peak is attributed to a large value of the Debye-Waller factor, which is further discussed in Section 5.3. 


\subsection{Zincblende phase}

In the $\mathrm{ZB}$ phase (below 3.65 $\mathrm{GPa}$ in the present experiment), the nearest-neighbour coordination number is 4 , independent of pressure. The quantitative analysis by the ratio method has allowed us to obtain the pressure dependence of the first three cumulants of the $\mathrm{Cd}$-Te distance distribution, say the cumulant values relative to the $100 \mathrm{kPa}$ reference.

Some examples of differences of phases and logarithms of amplitude ratios are given in figure 6. It is interesting to visually compare these plots with the corresponding plots of the temperature-dependent measurements on CdTe (figures 4 and 5 of [39]). In pressure-dependent measurements, the phase-difference lines are much more distant than in temperature-dependent measurements and nearly horizontal, owing to the much larger distance variations and to the much smaller variations of the third cumulant. By converse, the differences in the logarithms of amplitude ratios are smaller in pressuredependent measurements, owing to the small variations of the Debye-Waller factor.

The relative values of the cumulants from the ratio method were in very good agreement with the relative values obtained by the non-linear fitting of calculated to experimental EXAFS functions. An example of non-linear fitting in the real space is shown in figure 7.

In principle, the intrinsic inelastic factor $S_{0}^{2}$ depends on the interatomic distance, and hence on pressure. The pressure dependence could thus in principle affect the values of the second cumulant. The non-linear fit analysis didn't however reveal a definite trend of $S_{0}^{2}$ with pressure. Also the difference in the values of the second cumulant with respect to the analysis performed with $S_{0}^{2}$ set at the average value 1.05 had no definite trend and could be accounted for by a maximum uncertainty bar of the order of $5 \times 10^{-5} \AA^{2}$.

The absolute value of the nearest-neighbour Cd-Te distance at $294 \mathrm{~K}$ and $100 \mathrm{kPa}$ was evaluated by adding, to the crystallographic value $R_{c}=2.8052 \AA$ determined by diffraction measurements, the contribution due to the perpendicular MSRD previously obtained from the temperature-dependent EXAFS measurements [38]; the resulting value is $\langle r\rangle=2.816 \AA$. By considering this value as a fixed quantity in the nonlinear fitting procedure, an energy difference $e_{0}=4.015 \mathrm{eV}$ was found between the experimental and theoretical origins of the $k$ axis. The variation of the absolute distance as a function of pressure was then obtained by adding to $\langle r\rangle=2.816 \AA$ the relative values measured in the present experiment (right panel of figure 8).

Absolute values of the parallel MSRD (second cumulant) and of the third cumulant were evaluated by taking as reference the absolute values previously obtained at $294 \mathrm{~K}$ and $100 \mathrm{kPa}$ from temperature-dependent measurements [38] and adding the relative values here measured as a function of pressure (right panels in figures 10 and 11).

4.2.1. First cumulant: Bond compressibility The variations with temperature (at $100 \mathrm{kPa}$, data from [38]) and with pressure (at $294 \mathrm{~K}$, present work) of the first cumulant, corresponding to the bond distance, are shown in figure 8, where the EXAFS results are 


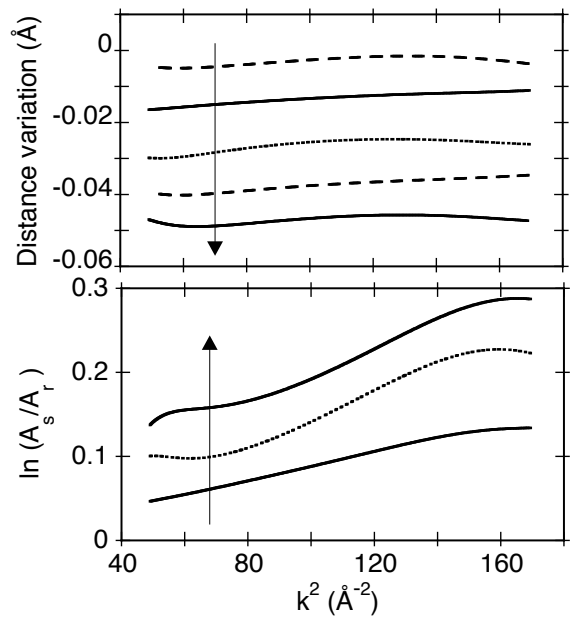

Figure 6. Differences of phases (top panel) and logarithms of the amplitudes ratios (bottom panel) at selected pressures $(0.46,1.1,1.85,2.6$ and $3.3 \mathrm{GPa}$ for phases, 1.1, 1.85 and $3.3 \mathrm{GPa}$ for amplitudes). The reference was the average of the $100 \mathrm{kPa}$ files measured at the end of the experiment. The lines are the average over the files measured at the same pressure. The arrows show the direction of increasing pressure.

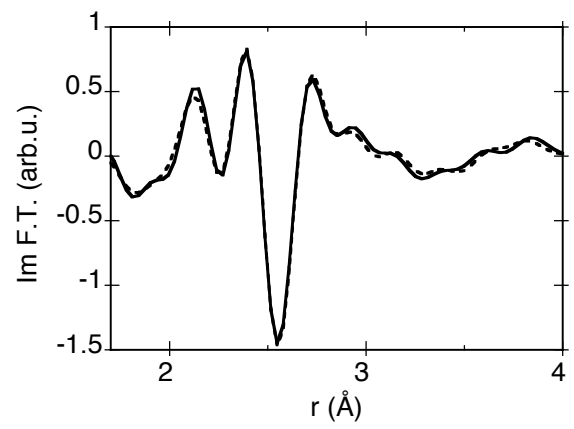

Figure 7. Comparison between the imaginary part of the Fourier transform at 1.1 $\mathrm{GPa}$ (continuous line) and the best fitting theoretical simulation (dashed line).
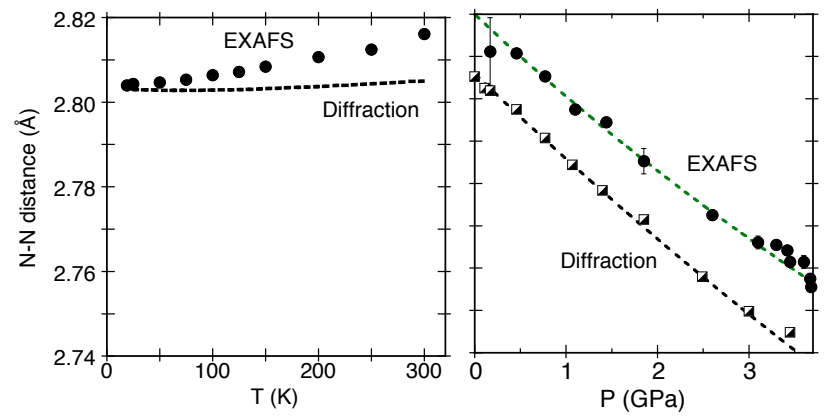

Figure 8. Bond distance Cd-Te measured by EXAFS and by diffraction as a function of temperature at $100 \mathrm{kPa}$ (left panel, data from [38]) and of pressure at $294 \mathrm{~K}$ (right panel, present work). The dashed lines in the right panel are the best-fitting Murnaghan EOS (see text). 
compared with the crystallographic values.

The bond thermal expansion measured by EXAFS (circles in the left panel) is larger than the expansion of the nearest-neighbour distance proportional to the lattice parameter $a$ measured by diffraction or by dilatometric techniques (dashed line in the left panel). The corresponding linear expansion coefficients above $100 \mathrm{~K}$ are $\alpha_{\text {bond }}=17 \times 10^{-6}$ and $\alpha_{\text {lat }}=5 \times 10^{-6} \mathrm{~K}^{-1}$, respectively [7]. A positive difference between the nearestneighbour distances and their expansions measured by EXAFS and by diffraction has been detected in all recently measured systems, and is attributed to the well known effect of relative atomic vibrations perpendicular to the bond direction [3, 58]. This effect is larger for CdTe [38] with respect to GaAs [59] and Ge [6], owing to the larger degree of ionicity, which reduces the rigidity of the interatomic bond.

As expected, when the pressure increases, the bond length measured at room temperature by both EXAFS and diffraction (circles and squares, respectively, in the right panel of figure 8) decreases; the pressure effect being by far more intense than the temperature effect, within the intervals of variation here considered. The EXAFS data point at $0.17 \mathrm{GPa}$ is affected by a large uncertainty bar, owing to the large differences between the data files measured at that pressure, probably due to the initial sample inhomogeneity. The uncertainty bars are much smaller at higher pressures, indicating a significant reduction of the sample inhomogeneity induced by pressure.

The unidimensional linear Murnaghan equation of state (EOS) [60]

$$
\ell(P)=\ell_{0}\left[P\left(\frac{K_{0}^{\prime}}{K_{0}}\right)+1\right]^{-1 /\left(3 K_{0}^{\prime}\right)}
$$

was fitted to the pressure dependence of the bond length measured by EXAFS and by diffraction $\left(\ell=\langle r\rangle\right.$ and $\ell=a \sqrt{3} / 4$, respectively); $K_{0}$ and $K_{0}^{\prime}$ are the isothermal bulk modulus and its first derivative, respectively, both evaluated at zero pressure.

The fitting procedure was limited to the pressure range 0.4 to $3.1 \mathrm{GPa}$, where both EXAFS and diffraction results exhibit a regular behaviour. Extending the fitting range to lower or higher pressures significantly modified the resulting parameters, particularly for EXAFS data. The deviations of the bond lengths measured by EXAFS from a regular behaviour can be due to poor sample homogeneity below $0.4 \mathrm{GPa}$ (at the beginning of the experiment) and to a small contribution of the cinnabar phase above $3.1 \mathrm{GPa}$. The best-fitting Murnaghan EOS are shown as dotted lines in the right panel of figure 8 . The fitting parameters are listed and compared with other works in table 1 , where the zero-pressure one-dimensional isothermal compressibilities

$$
\chi_{0}^{\operatorname{lin}}=-\frac{1}{\ell}\left(\frac{\partial \ell}{\partial P}\right)_{T}=\frac{1}{3 K_{0}}
$$

are listed too.

The zero-pressure bulk modulus $K_{0}$ from EXAFS is slightly larger than the value from diffraction, corresponding to a slightly smaller zero-pressure compressibility $\chi_{0}^{\operatorname{lin}}$. Much stronger are the differences between the values of the zero-pressure first derivatives $K_{0}^{\prime}=(d K / d P)_{0}$ measured by EXAFS and diffraction. 
Table 1. The isothermal bulk moduli $K_{0}$, their first derivatives $K_{0}^{\prime}$ and the onedimensional isothermal compressibilities $\chi_{0}^{\text {lin }}$ evaluated at zero pressure from EXAFS and diffraction results through a fit to the Murnaghan equation of state (1).

\begin{tabular}{lccc}
\hline & $K_{0}(\mathrm{GPa})$ & $K_{0}^{\prime}$ & $\chi_{0}^{\operatorname{lin}}\left(10^{-12} \mathrm{~Pa}^{-1}\right)$ \\
\hline EXAFS (present) & 45.9 & 4.7 & 7.23 \\
Diffraction (present) & 44.8 & 2.7 & 7.4 \\
Diffraction (Ref. [42]) & 43.7 & 3.8 & 7.6 \\
Theory (Ref. [44]) & 46.0 & & \\
\hline
\end{tabular}

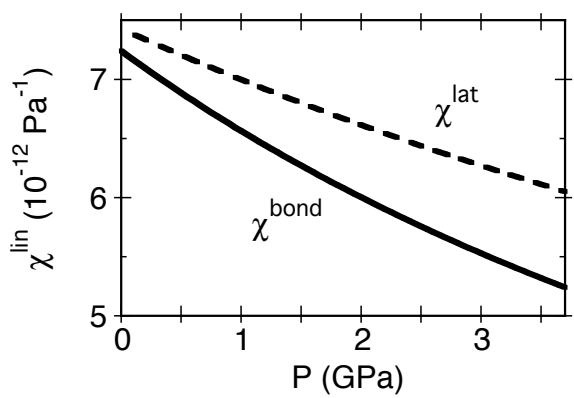

Figure 9. Isothermal bond compressibility measured by EXAFS (continuous line) and lattice compressibility measured by diffraction (dashed line).

The isothermal linear compressibilities $\chi^{\operatorname{lin}}(P)$ as a function of pressure have been calculated from the first derivatives of the best-fitting EOS (1) and are shown in figure 9. As expected, the compressibilities decrease as the pressure increases; the decrease is significantly stronger for the bond compressibility $\chi^{\text {bond }}(P)$ measured by EXAFS than for the lattice compressibility $\chi^{\text {lat }}(P)$ measured by diffraction. This result is discussed in Section 5.1.

4.2.2. Second and third cumulants The second cumulant $C_{2}$, say the variance of the distribution of $\mathrm{Cd}-\mathrm{Te}$ distances (parallel MSRD) is shown in figure 10. The third cumulant $C_{3}$, which monitors the asymmetry of the distribution, is shown in figure 11 . In both figures, the left panel refers to the temperature dependence at the constant $100 \mathrm{kPa}$ pressure [38], the right panel refers to the present measurements as a function of pressure, at the constant temperature $T=294 \mathrm{~K}$.

Let us first review the temperature-dependent behaviour [10, 38]. As a first approximation, the second cumulant can be fitted to an Einstein model whose sole parameter is the frequency $\nu_{E}$ and the third cumulant can be fitted to a quantum perturbative model $[61,62]$

$$
C_{3}^{*} \simeq-\frac{2 k_{3} \sigma_{0}^{4}}{k_{0}} \frac{z^{2}+10 z+1}{(1-z)^{2}}
$$

where the quantities $z=\exp \left(-h \nu / k_{B} T\right), k_{0}=4 \pi^{2} \nu^{2} \mu$ and $\sigma_{0}^{2}=h / 4 \pi^{2} \nu \mu$ can be evaluated from the Einstein frequency; $\mu$ is the reduced mass. A third-order force 
constant $k_{3}=-2.12 \pm 0.03 \mathrm{eV} \AA^{-3}$ of the effective pair potential was obtained from the fit of (3) to the experimental data.

A more refined analysis of the temperature-dependent data based on the knowledge of the third and fourth cumulants allowed the separation of the harmonic and anharmonic contributions to the second cumulant [10]: the harmonic contribution (dashed line in the left panel of figure 10) corresponds to a constant Einstein frequency $\nu_{0}=4.05 \mathrm{THz}$, say to a harmonic force constant $k_{0}=4.01 \mathrm{eV} / \AA^{2}$; the anharmonic contribution increases with temperature, so that the Einstein frequency best fitting the total $C_{2}$ (continuous line in the left panel of figure 10) decreases from $4.05 \mathrm{THz}$ at $0 \mathrm{~K}$ to $3.87 \mathrm{THz}$ at $294 \mathrm{~K}$, corresponding to a decrease of the effective force constant $k$ from 4.01 to $3.66 \mathrm{eV} / \AA^{2}$.

Let us now consider the cumulants as a function of pressure (right panels in figures 10 and 11). The absolute values of both second and third cumulants at $100 \mathrm{kPa}$ have been set equal to the values obtained from the temperature dependent measurements at $294 \mathrm{~K}$.

When the pressure increases, the second cumulant decreases (circles in the right panel of figure 10). This reduction has no structural contribution, since no static disorder was present even at $100 \mathrm{kPa}$. Actually, the decrease of the Debye-Waller factor of both diffraction and EXAFS when the bond distance decreases is expected on purely vibrational grounds, as an effect of anharmonicity $[58,16]$; the topic is further discussed in Section 5.3. The Einstein force constant increases from $k=3.66 \mathrm{eV} / \AA^{2}$ at $100 \mathrm{kPa}$ to $4.15 \mathrm{eV} / \AA^{2}$ at $3.5 \mathrm{GPa}$.

The values of the third cumulant as a function of pressure are quite scattered (right panel in figure 11), in comparison with the temperature dependent measurements, reflecting a higher degree of uncertainty of experimental data. A slight reduction, from about $1.5 \times 10^{-4}$ to about $1.3 \times 10^{-4} \AA^{3}$, can be observed when the pressure increases from $100 \mathrm{kPa}$ to $3.5 \mathrm{GPa}$. This reduction has been tentatively fitted to a linear behaviour (dashed line). The behaviours of the second and third cumulants of CdTe are qualitatively similar to those observed in solid $\mathrm{Kr}$ [19].

In order to estimate the harmonic contribution to the second cumulant in pressure-dependent measurements, we made use of the approximate classical expressions connecting the cumulants to the force constants of the effective potential [63] and neglected the contribution of the fourth cumulant:

$$
\begin{aligned}
& C_{2} \simeq \frac{k_{B} T}{k_{0}}+\frac{36 k_{3}^{2}}{k_{0}^{4}}\left(k_{B} T\right)^{2} \\
& C_{3} \simeq-\frac{6 k_{3}}{k_{0}^{2}}\left(k_{B} T\right)^{2},
\end{aligned}
$$

where $T=294 \mathrm{~K}$. The result is shown in the right panel of figure 10, where the circles represent the total $C_{2}$ values (the continuous line is a parabolic fit) and the dashed line represents the harmonic contribution. When the pressure increases from $100 \mathrm{kPa}$ to 3.5 GPa, the classical total and harmonic effective force constants increase of 15 and 17 percent, respectively. The classical approximation underestimates the force constants 


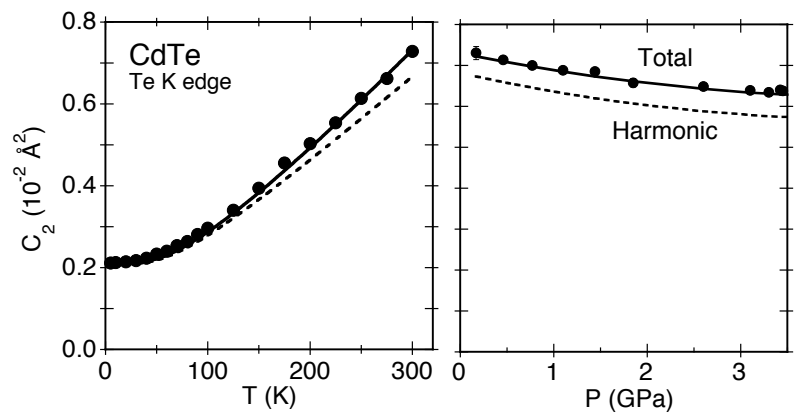

Figure 10. Second cumulant of the Cd-Te distribution as a function of temperature at $p=100 \mathrm{kPa}$ (left panel, data from [10]) and as a function of pressure at $T=294 \mathrm{~K}$ (right panel, present work). The circles are experimental points, the continuous lines are the parabolic best fits to their behaviour, the dashed lines are the harmonic contributions.

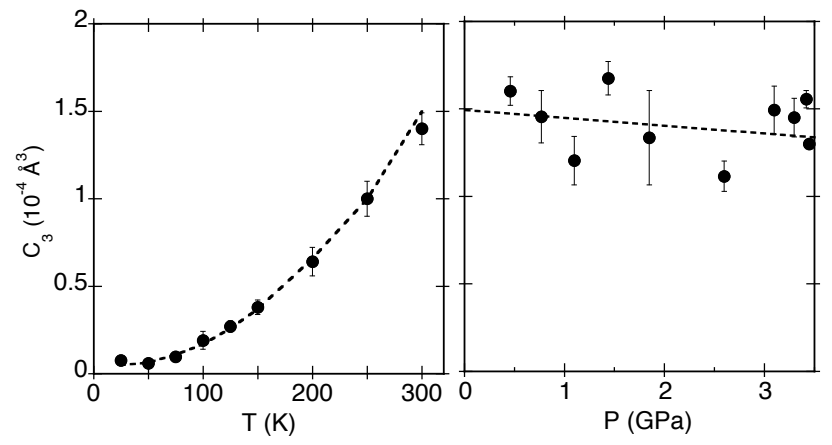

Figure 11. Third cumulant of the Cd-Te distribution. Left panel: as a function of temperature at $p=100 \mathrm{kPa}$ (data from [38]), the dashed line is the best fit according to equation (3). Rigth panel: as a function of pressure at $T=300 \mathrm{~K}$ (present work); the dashed line is a linear best fit.

with respect to the quantum values; it is however reasonable to assume that the relative variation with pressure is the same for the classical and quantum force constants, if the temperature is not varied. This assumption leads to an increase of the harmonic force constant $k_{0}$ from 4.01 at $100 \mathrm{kPa}$ to $4.7 \mathrm{eV} / \AA^{2}$ at $3.5 \mathrm{GPa}$.

\subsection{Mixed cinnabar-zincblende phase}

When increasing the pressure at room temperature, McMahon et al. [42] first detected a cinnabar-like phase mixed to ZB in diffraction patterns at about $3.5 \mathrm{GPa}$; at $3.8 \mathrm{GPa}$ the transition to the pure RS structure was nearly complete. The pressure range in which the cinnabar phase is observed decreases when the temperature increases, so that at $773 \mathrm{~K}$ the transition directly occurs between the ZB and the RS phases [64].

In the present work, the mixed ZB-cinnabar phase was observed in the pressure range from 3.7 to $4.0 \mathrm{GPa}$ (Section 4.1), the cinnabar volume fraction increasing from 
12 to $44 \%$, according to the refinement of diffraction patterns.

According to McMahon et al. [42], in the cinnabar-like phase each $\mathrm{Cd}$ atom is coordinated to six Te atoms, at three different distances, which exhibit a different pressure dependence: at about $3.5 \mathrm{GPa}$, two nearest neighbours are at 2.745 , two at 2.948 and two at $3.441 \AA$, respectively. In the mixed phase ZB-cinnabar there are thus four different $\mathrm{Cd}$-Te bond lengths, with corresponding different Debye-Waller factors and third cumulants. A complete un-constrained EXAFS characterization of the local structure is out of question, in view of the exceedingly large number of free parameters involved. One cannot thus expect to obtain original results from the EXAFS analysis. We only checked that the experimental EXAFS spectra are in good agreement with the spectra simulated starting from the crystallographic values of distances.

\subsection{Rocksalt phase}

We consider here the results of measurements performed on the RS phase at $4.75 \mathrm{GPa}$. The lattice parameter obtained from diffraction is $a=5.8943 \AA$, corresponding to a crystallographic nearest-neighbour distance $R_{c}=2.947 \AA$.

The analysis by the ratio method of the three EXAFS spectra measured at $4.75 \mathrm{GPa}$ was made by taking the $\mathrm{ZB}$ spectrum at $100 \mathrm{kPa}$ as reference. The phase analysis gives a Cd-Te distance of $2.933 \AA$ and a third cumulant of $4.9 \times 10^{-4} \AA^{3}$, significantly larger than the values of the $\mathrm{ZB}$ phase. The amplitude analysis, performed taking into account the difference in bond distance between the ZB and the RS phases and considering both the coordination number and the second cumulant as fitting parameters, gives a coordination number $\simeq 4.9$ and a second cumulant $C_{2} \simeq 0.012 \AA^{2}$. If the coordination number is fixed to the expected value 6 , the quality of the fit significantly worsens and the second cumulant increases to $0.013 \AA^{2}$.

The non-linear fit to simulated spectra was made by assuming $e_{0}=4.015 \mathrm{eV}$ and $S_{0}^{2}=1.05$, as in the ZB phase. One obtains the correct coordination number 6 , a second cumulant $C_{2} \simeq 0.013 \AA^{2}$, a distance $\langle r\rangle=2.943 \AA$ and a third cumulant $C_{3} \simeq 8 \times 10^{-4} \AA^{3}$.

We consider the ratio method less reliable than the non-linear fit in the case of the RS phase, since it gives a coordination number 4.9, smaller than the expected value 6 , and a $\mathrm{Cd}$-Te distance more than $0.01 \AA$ shorter than the crystallographic distance (while it is expected to be larger owing to the effect of perpendicular vibrations). The inadequacy of the ratio method can be attributed to the assumption of the same backscattering amplitudes and phaseshifts for two significantly different structures (the $\mathrm{RS}$ and the ZB reference, respectively). The non linear fit procedure gives instead the correct coordination number; however, the $\mathrm{Cd}$-Te distance is still slightly shorter than the crystallographic distance; this last discrepancy could be due to the wrong assumption of a same $e_{0}$ value for the two phases, ZB and RS.

In any case, both procedures agree on a significantly larger value of the second cumulant in the RS phase $\left(C_{2} \simeq 0.013 \AA^{2}\right.$ at $\left.4.75 \mathrm{GPa}\right)$ than in the $\mathrm{ZB}$ phase 
$\left(C_{2} \simeq 0.007 \AA^{2}\right.$ at $\left.100 \mathrm{kPa}\right)$. A similar difference was previously observed in $\mathrm{ZnTe}$ and tentatively attributed to a distortion of the first-shell octahedron in the rosksalt phase, say to a structural origin [18]. Actually, as already observed for the ZB phase, an increase of the second cumulant when the bond distance increases is expected even on purely vibrational grounds, as an effect of anharmonicity [16]. This topic is further discussed at the end of Section 5.3.

\section{Discussion}

\subsection{Bond and lattice compressibilities}

According to the present measurements, the bond compressibility in CdTe is slightly smaller than the lattice compressiblity, and decreases significantly faster when the pressure increases (figure 9).

A slightly lower compressibility was found also by San-Miguel et al. in ZnTe from EXAFS with respect to diffraction, but the difference was attributed to experimental and data analysis uncertainties [18]. More recently, Pellicer-Porres et al. studied the bond length compressibility of GaSe [21] and of hard $\mathrm{ReB}_{2}$ [23] by dispersive XAFS. In the low-pressure phase of GaSe, the Ga-Se bond compressibility determined from EXAFS was $\chi_{\mathrm{b}}=(3.6 \pm 0.2) \times 10^{-12} \mathrm{~Pa}^{-12}$, smaller than the linear lattice compressibilities along the $a$ and $c$ axes, $\chi_{a}=5 \times 10^{-12}$ and $\chi_{c}=24.9 \times 10^{-12} \mathrm{~Pa}^{-12}$, respectively. In $\mathrm{ReB}_{2}$, the Re-B bond compressibility was smaller than the lattice compressibility, which in turn was smaller than the B-B bond compressibility.

In view of the experience gained from temperature-dependent measurements, we suggest a physical origin to the different behavior of bond and lattice compressibilities. When the pressure increases, both the distance between the average atomic positions $R_{c}$, measured by diffraction, and the bond length $\langle r\rangle$, measured by EXAFS, decrease. The direction of $\vec{R}_{c}$ is constrained by symmetry, the instantaneous direction of $\vec{r}$ is determined by the instantaneous direction and extent of the perpendicular relative displacement $\Delta \vec{u}_{\perp}$. One well known consequence is that $\langle r\rangle$ is larger than $R_{c}[3,10]$. It is reasonable that, when the pressure increases, the balance between parallel and perpendicular vibrational energy is modified so that the parallel contribution is decreased and the perpendicular contribution is increased (see also Section 5.2.2). As a consequence, the reduction of the bond distance is progressively smaller than the reduction of the crystallographic distance (proportional to the lattice parameters), so that the bond compressibility becomes progressively smaller than the lattice compressibility.

\subsection{Distributions of distances and effective pair potential}

5.2.1. Unidimensional distribution EXAFS samples a unidimensional distribution of bond distances. Within the range of validity of the cumulant expansion method, typically for relatively weak vibrational disorder, the distribution can be reconstructed with good accuracy from the knowledge of the leading cumulants $[9,51]$. The real 


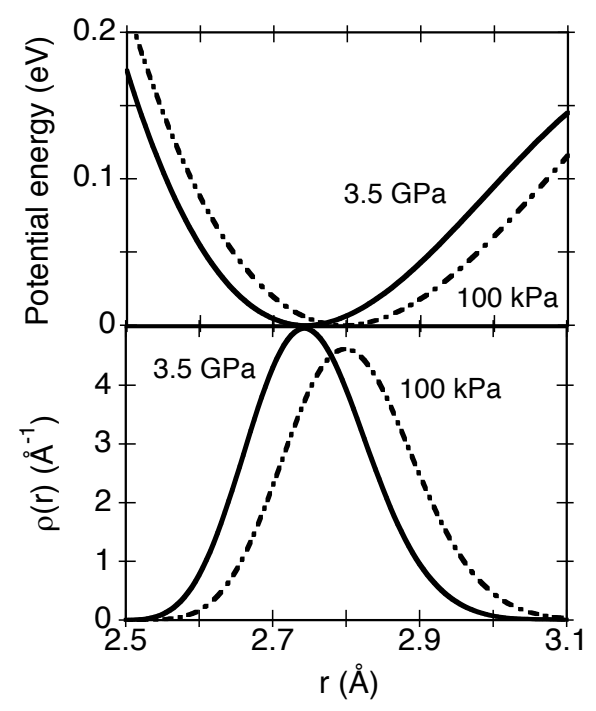

Figure 12. Effective pair potentials (top panel) and corresponding real distributions (bottom panel) reconstructed from the EXAFS cumulants at $100 \mathrm{kPa}$ (thick dashdotted lines) and at 3.5 GPa (thick continuous lines).

distributions $\rho(r)$ reconstructed from the cumulants for the ZB phase of CdTe at the two extreme pressures of $100 \mathrm{kPa}$ and $3.5 \mathrm{GPa}$ are shown in the bottom panel of figure 12 . When the pressure increases, the reduction of the bond distance (figure 8), say of the mean value, is accompanied by a reduction of the mode of the distribution. Second and third cumulants slightly decrease when the pressure increases, but the asymmetry parameter of the distribution, conventionally defined as [65] $C_{3} / C_{2}^{3 / 2}$, slightly increases from about 0.245 to about 0.274 .

The complex relationships between force constants, cumulants and asymmetry parameter is discussed on general grounds in [9]. We suggest here that the slight increase of asymmetry when the pressure increases can be somewhat connected to the perpendicular vibrations and in particular to the increase of vibrational anisotropy (to be further discussed in Section 5.2.2), which would progressively enhance the contribution of the larger distances to the distribution.

One frequently associates the distribution of distances with an effective pair potential

$$
V(x)=\frac{1}{2} k_{0} x^{2}+k_{3} x^{3}+k_{4} x^{4},
$$

where $x=r-r_{0}$ is the deviation of the distance from the minimum position, which corresponds to the mode of the distribution. The lowest-order force constants $k_{i}$ of the effective pair potential can be derived from the knowledge of the leading cumulants $[63,61,62,39]$.

The effective pair potential is in principle dependent on temperature and pressure. In constant-pressure measurements as a function of temperature, the effective force constants $k_{i}$ can be generally considered as independent of temperature [39]; a rigid 
shift of the effective potential has anyway to be taken into account, which significantly contributes to the bond expansion measured by the first cumulant [9].

In the present constant-temperature measurements on CdTe as a function of pressure, one finds not only a significant shift of the mode of the distribution, corresponding to a shift of the minimum position of the effective pair potential, but even a non negligible variation of the second and third-order force constants, $k_{0}$ and $k_{3}$, respectively (Section 4). When the pressure increases, the effective potential becomes harder (the harmonic force constant increases); also the anharmonicity ratio $\left|k_{3}\right| / k_{0}$ increases, from about 0.67 to about $0.85 \AA^{-1}$. No significant variation of the 4 th cumulant is instead detected with respect to the value $k_{4}=-0.36 \mathrm{eV} / \AA^{4}$ previously measured at $294 \mathrm{~K}$ and $100 \mathrm{kPa}$ [39].

The effective pair potentials $V(r)$ calculated for CdTe in the ZB structure at the two limiting pressures $100 \mathrm{kPa}$ and $3.5 \mathrm{GPa}$ are shown in the upper panel of figure 12 . The negative values of $k_{3}$ correspond to a softening of the attractive part and a hardening of the repulsive part of the potential with respect to the harmonic potential; the negative value of $k_{4}$ gives rise to a very weak symmetric softening of the potential.

The main visible effect of increasing the pressure is the nearly rigid shift to lower distances of both the effective potential and the distribution. The variations of $k_{0}$ and $k_{3}$ only slightly modify the shape of the effective potential and, as a consequence of thermal vibrations, of the corresponding distribution.

5.2.2. Three-dimensional distribution and perpendicular MSRD The parallel MSRD $\left\langle\Delta u_{\|}^{2}\right\rangle=C_{2}$ is directly obtained from the EXAFS analysis. For the ZB phase, we found that the parallel MSRD decreases from 0.0072 to $0.0062 \AA^{2}$ when the pressure increases from $100 \mathrm{kPa}$ to $3.5 \mathrm{GPa}$. The perpendicular MSRD $\left\langle\Delta u_{\perp}^{2}\right\rangle$ can be evaluated from the difference between the bond distances $\langle r\rangle$ and $R_{c}$ measured by EXAFS and by diffraction, respectively [10]:

$$
\left\langle\Delta u_{\perp}^{2}\right\rangle=2 R_{c}\left[\langle r\rangle-R_{c}\right] .
$$

The difference between the two values $\langle r\rangle$ and $R_{c}$ of bond distance increases when the pressure increases (figure 8, right panel); correspondingly, the perpendicular MSRD increases: its projection along one direction $\left\langle\Delta u_{\perp}^{2} / 2\right\rangle$ increases from 0.032 to $0.047 \AA^{2}$ when the pressure increases from $100 \mathrm{kPa}$ to $3.5 \mathrm{GPa}$.

The knowledge of both parallel and perpendicular MSRDs allows one to reconstruct the three-dimensional distribution of instantaneous vector distances $\vec{r}$ between absorber and backscatterer atoms [9]. A schematic representation of the ellipsoids of relative vibration for the ZB phase of CdTe at the two extreme pressures $100 \mathrm{kPa}$ and $3.5 \mathrm{GPa}$ is shown in Fig. 13. The longitudinal and transverse axes are proportional to $\sigma_{\|}=\left[\left\langle\Delta u_{\|}^{2}\right\rangle\right]^{1 / 2}$ and to $\sigma_{\perp}=\left[\left\langle\Delta u_{\perp}^{2}\right\rangle / 2\right]^{1 / 2}$, respectively. As effect of lattice compression, the intensity of relative vibrations is reduced along the bond direction and enhanced in perpendicular direction, so that their anisotropy increases.

It is interesting to compare EXAFS and diffraction results on the intensities of 

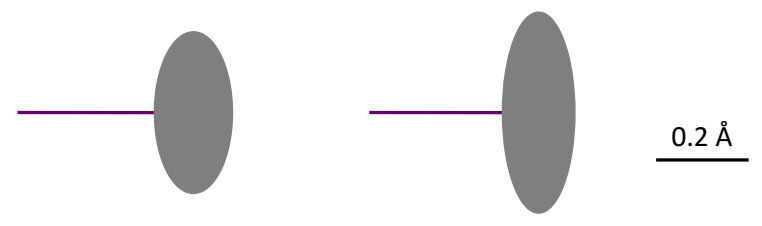

Figure 13. Two-dimensional projection of the ellipsoids of thermal vibration evaluated at $100 \mathrm{kPa}$ (left) and at $3.5 \mathrm{GPa}$ (right). The horizontal lines show the bond direction.

atomic vibrations. The atomic displacements in the $\mathrm{ZB}$ phase of $\mathrm{CdTe}$ are expected to be isotropic for symmetry reasons. Unfortunately, the accuracy of the isotropic displacement parameters obtained in the present experiment is not sufficient to detect a well defined trend as a function of pressure nor to allow a meaningful comparison with the EXAFS MSRDs. We can only rely on the results of accurate diffraction measurements performed at ambient pressure [66], according to which the sum of the mean square displacements of $\mathrm{Cd}$ and Te along the bond direction is $0.0394 \AA^{2}$ at room temperature. The comparison of those diffraction data with the MSRDs from EXAFS at $100 \mathrm{kPa}$ revealed a strong correlation for parallel vibrations and a weak correlation for perpendicular vibrations [38]; actually, the different degree of correlation is responsible for the anisotropy of relative vibrations. If we assume a negligible or negative variation of the atomic displacement parameters when the pressure increases, the value of perpendicular MSRD at 3.5 GPa would imply a slightly negative correlation (say vibrations in slight opposition of phase) in the perpendicular direction.

Let us now try to better understand the origin of the different behaviour of parallel and perpendicular MSRDs as a function of pressure. The MSRDs are the sum of the contributions of all normal modes of the crystal [2,67]; different normal modes can differently contribute to the parallel and perpendicular MSRD, giving rise to the anisotropy of relative vibrations, which is different for different crystals [9]. At constant pressure, the ratio $\left\langle\Delta u_{\perp}^{2}\right\rangle /\left\langle\Delta u_{\|}^{2}\right\rangle$ is dependent on temperature only at low temperatures, where the populations of different normal modes are differently changing with temperature; at high temperatures, in the classical limit, the ratio and the corresponding anisotropy become independent of temperature [8].

At constant temperature, when the pressure is changed, the frequency of normal modes is changed too; the change of frequency can be quantified by the mode GPs and their pressure dependence. In many ZB structures, such as CdTe, the large majority of transverse acoustic (TA) modes are characterized by negative GPs [68, 69], whose value further decreases when the pressure increases [70]. TA modes give rise to rotations of the tetrahedra of first-shell coordination [69] and are responsible for the low temperature negative lattice thermal expansion. It is reasonable to assume that TA modes significantly contribute to the perpendicular MSRD and give rise to its enhancement when the pressure increases. 
It is interesting to notice that an increase of anisotropy of atomic displacement parameters has been detected by neutron powder diffraction on lead scandium niobate, where the oxygen parameters increase along the $\langle 110\rangle$ directions and decrease along the $\langle 001\rangle$ directions when the pressure increases [71].

\subsection{Anharmonicity and Grüneisen parameters}

A well known effect of anharmonicity for a single oscillator is the variation of the frequency with the amplitude of oscillations [72]. For a thermodynamic system, such as a crystal, the relation between frequency and volume or between frequency and a linear distance (be it a lattice parameter or a bond length) can be defined in different ways, not necessarily within the QHA approximation which underpins the elementary theory of thermal expansion [12]. Notice that the linear Grüneisen parameters (GP) are always three times larger than the corresponding volume GPs.

EXAFS experiments typically give the variation of the bond distance $\langle r\rangle$ and of the second cumulant as a function of temperature (at constant pressure) or of pressure (at constant temperature); the values of the second cumulant can in turn be connected to the frequency $\nu$ of the best-fitting Einstein model. One can thus define and evaluate a linear bond GP

$$
\gamma_{\mathrm{b}}=-\frac{\langle r\rangle}{\nu}\left(\frac{\partial \nu}{\partial\langle r\rangle}\right)
$$

which can assume different values according to whether the derivative is calculated at constant pressure or at constant temperature.

5.3.1. Constant-pressure bond Grüneisen parameter Let us begin our discussion from the results of the previous EXAFS study of CdTe as a function of temperature [10], where the harmonic and anharmonic contributions to the second cumulant were singled out (left panel in figure 10). The temperature-dependent frequency $\nu(T)$ was obtained by fitting an Einstein model to the second cumulant at each temperature; its values are plotted as a function of the reduced bond distance in figure 14 (squares). From the logarithmic derivative of the frequency with respect to the bond distance, a constantpressure bond GP $\gamma_{\mathrm{b}, p} \simeq 9$ was obtained, with no significant dependence on temperature from 50 to $300 \mathrm{~K}$. It is worth remembering that the thermal bond expansion is determined by both the anharmonicity and the rigid shift of the effective pair potential [9]. If only the contribution to the bond expansion due to the potential anharmonicity is considered[38], the bond GP increases to $\tilde{\gamma}_{\mathrm{b}, p} \simeq 11$.

It is instructive to compare the experimental values of the bond GP with the values calculated from the lowest-order classical expression of the cumulants [63] as a function of only the second and third-order force constants $k_{0}$ and $k_{3}$ :

$$
\begin{aligned}
\delta \tilde{C}_{1}^{*}(T) & \simeq-\frac{3 k_{3}}{k_{0}^{2}} k_{B} T \\
C_{2}^{*}(T) & \simeq \frac{k_{B} T}{k_{0}}+\frac{36 k_{3}^{2}}{k_{0}^{4}}\left(k_{B} T\right)^{2} \simeq \frac{k_{B} T}{k} .
\end{aligned}
$$




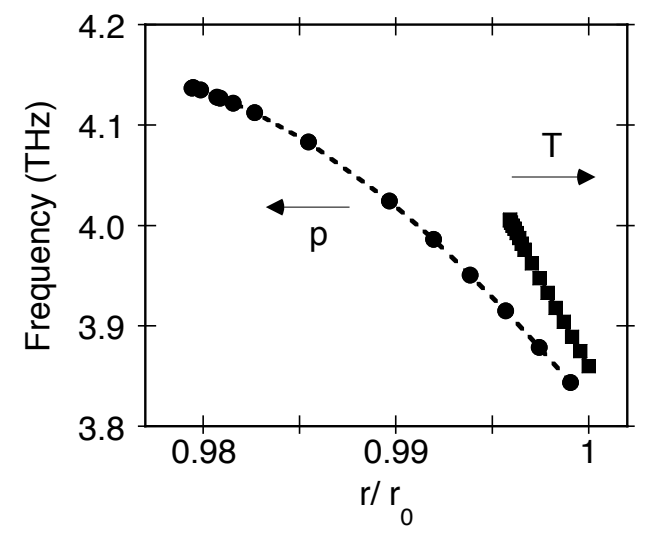

Figure 14. Einstein frequency as a function of the reduced bond distance for $T$ dependent (squares) and $p$-dependent (circles) EXAFS measurements. The arrows give the direction of pressure or temperature increase.

Here $\delta \tilde{C}_{1}^{*}(T)=\delta\langle\tilde{r}\rangle$ is the contribution to the bond expansion only due to the potential anharmonicity (no contribution from potential shift) and $k$ is the temperature-dependent force constant of the Einstein model best fitting at each temperature. The corresponding frequency $\nu$ is connected to the harmonic frequency $\nu_{0}$ by

$$
\nu(T) \simeq \nu_{0}\left[1-\left(\frac{36 k_{3}^{2}}{k_{0}^{3}}\right) k_{B} T\right]^{1 / 2} \simeq \nu_{0}\left[1+\frac{12 k_{3} \delta \tilde{C}_{1}^{*}}{k_{0}}\right]^{1 / 2}
$$

from which one finds a constant-pressure linear GP

$$
\tilde{\gamma}_{\mathrm{b}, p}(T) \simeq-\frac{6 k_{3} C_{1}^{*}}{k_{0}} \frac{1}{1+12 k_{3} \delta C_{1}^{*} / k_{0}} .
$$

By inserting into (12) the experimental values of $k_{0}, k_{3}, C_{1}^{*}$ and $\delta C_{1}^{*}$ from [38], one obtains $\tilde{\gamma}_{\mathrm{b}, p} \simeq 10$ at $300 \mathrm{~K}$. This value is intermediate between the values 9 and 11 obtained from the logarithmic derivative of the frequency with respect to the full expansion and the expansion solely due to the effective potential anharmonicity, respectively.

It is interesting to note that from a quasi-harmonic approach to the single oscillator [61] or to a linear chain[72] one obtains a linear GP two times smaller than in (12):

$$
\tilde{\tilde{\gamma}} \simeq-\frac{3 k_{3}\langle\tilde{r}\rangle}{k_{0}}
$$

This discrepancy suggests that the quasi-harmonic approximation, where only the harmonic oscillations around the thermally expanded average bond are considered, is insufficient to account for the full anharmonic contribution to the second cumulant.

5.3.2. Constant-temperature bond Grüneisen parameter The Einstein frequencies best fitting the total parallel MSRDs (circles in the right panel of figure 10) are shown as a function of the reduced bond length in figure 14 (circles). The deviation from a linear 
behaviour is evident for the $p$-dependent measurements, where the relative variation of the bond length is much larger than in $T$-dependent measurements.

From the logarithmic derivative of the frequency with respect to the bond length, one obtained the values of the constant- $T$ bond GP $\gamma_{\mathrm{b}, T}$, which are shown in the left panel of figure 15. The constant- $T$ bond GP progressively decreases from about 6 at $100 \mathrm{kPa}$ to about 1.8 at $3.5 \mathrm{GPa}$. The dependence of the constant-temperature bond GP $\gamma_{\mathrm{b}, T}$ on the reduced bond distance $r / r_{0}$ is shown in the right panel of figure 15 .

The reduction of $\gamma_{\mathrm{b}, T}$ of CdTe when the pressure increases is much faster than the one observed for the thermodynamic GP in metals $[73,74,75]$ and in noble gas solids [76] and is not compatible with the nearly linear relation $\gamma \simeq \gamma_{0}\left(V / V_{0}\right)$ generally assumed for those systems. Faster than in close-packed structures is the decrease of the mode GPs of a number of tetrahedral compounds with the ZB structure [70]; in those systems, some mode GPs are negative at room pressure or become progressively negative when the pressure increases.

One can reasonably expect that the mode GPs of the metallic close-packed and of the relatively open $\mathrm{ZB}$ structures have a different dependence on pressure. In the present case, we are considering bond GPs, whose relationship with the mode and thermodynamic GPs is far from trivial (see below). The strong reduction of the constant-temperature bond GP observed in the present experiment is anyway related to the reduction of the mode GPs; in particular, the proportion and weight of modes with negative GP increase with respect to modes with positive GP when the pressure increases, giving rise to the growth of the anisotropy of relative atomic vibrations. The increase of vibrational anisotropy could in turn contribute to the instability of the ZB phase which leads to the transition from ZB to cinnabar.

Let us now compare the constant-pressure and constant-temperature bond GPS evaluated from temperature-dependent and pressure-dependent EXAFS measurements. In the same conditions of temperature and pressure $(294 \mathrm{~K}$ and $100 \mathrm{kPa}$, corresponding to the bottom-right corner of figure 14$), \gamma_{\mathrm{b}, T} \simeq 6$ is significantly smaller than $\gamma_{\mathrm{b}, p} \simeq 9$. When the pressure increases at constant temperature, $\gamma_{\mathrm{b}, T}$ significantly decreases, whereas when the temperature decreases at constant pressure the variations of $\gamma_{\mathrm{b}, p}$ are negligible. Qualitatively, a temperature reduction (at constant pressure) and a pressure increase (at constant temperature) produce similar effects: a reduction of bond distance and an increase of Einstein frequency (which in turn reflects the increase of effective force constant). The relation between variation of frequency and variation of distance is however quantitatively different in the two cases. As a matter of fact, from figures 14 and 8 one can see that the same increase of frequency from 3.86 to $4 \mathrm{THz}$ is accompanied by a decrease of distance of $0.016 \AA$ when the temperature is reduced from $294 \mathrm{~K}$ to $5 \mathrm{~K}$ and by a decrease of $0.04 \AA$ when the pressure is increased from $100 \mathrm{kPa}$ to $1.57 \mathrm{GPa}$. This different behavior could be explained by considering that the variation of bond distance when the temperature is varied at constant pressure is a purely thermal effect induced by anharmonicity, while the variation of bond distance when the pressure is varied at constant temperature involves also the effect of external mechanical work. 

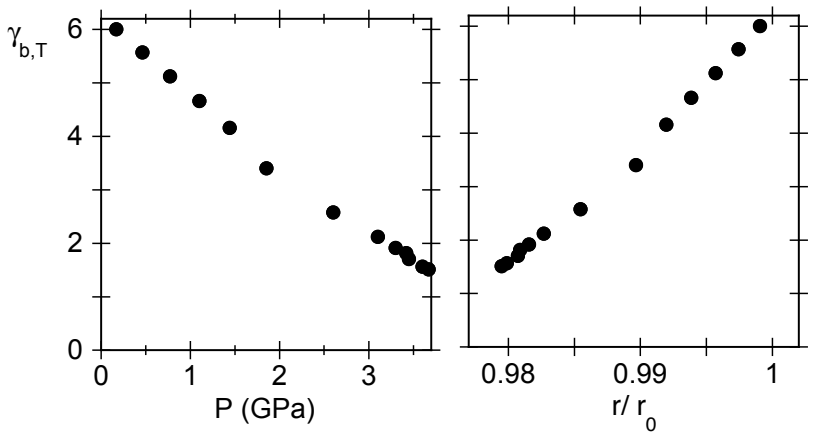

Figure 15. Bond Grüneisen parameter at the constant temperature $T=294 \mathrm{~K}$ as a function of pressure (left) and reduced bond distance (right) in the ZB phase of CdTe.

5.3.3. Relation with mode Grüneisen parameters The coefficient of volumetric thermal expansion can be expressed as

$$
\beta=\frac{1}{V}\left(\frac{\partial V}{\partial T}\right)_{p}=\chi_{T} \frac{C_{v}}{V} \gamma_{v}
$$

where $\chi_{T}$ is the isothermal volume compressibility, $c_{v}$ is the constant-volume molar specific heat and $\gamma_{v}$ is the thermodynamic GP, which, within the QHA, can be expressed as

$$
\gamma_{v}(T)=\frac{\sum_{\vec{q} s} c_{v}(\vec{q} s) \gamma(\vec{q} s)}{\sum_{\vec{q} s} c_{v}(\vec{q} s)},
$$

say as a weighted average of the (constant-temperature) mode GPs [12]

$$
\gamma(\vec{q} s)=-\left(\frac{\partial \ln \nu(\vec{q} s)}{\partial \ln V}\right)_{T}
$$

It is interesting to investigate the possible relation between the constanttemperature bond GP $\gamma_{\mathrm{b}, T}$ determined from the pressure-dependent EXAFS measurements and the mode GPs. To that purpose, let us consider the classical approximation to the Einstein model, which is sufficient for CdTe at room temperature.

The second cumulant can be expressed as

$$
C_{2}=B \sum_{i} \frac{A_{i}}{\nu_{i}^{2}}=\frac{B}{\nu_{E}^{2}},
$$

where the second member contains the sum over the normal modes, the third term approximates the second cumulant by a single Einstein frequency, and

$$
B=\frac{k_{B} T}{4 \pi^{2} \mu}, \quad A_{i}=\left|\left(\vec{w}_{b i}-\vec{w}_{a i}\right) \cdot \hat{R}\right|^{2}
$$

where $\vec{w}$ are the normalised mode eigenvectors. The logarithmic derivative of the Einstein frequency with respect to a linear distance $\ell$ is

$$
\frac{d \ln \nu_{E}}{d \ln \ell}=\frac{1}{\sum_{i} A_{i} \nu_{i}^{-2}}\left[\sum_{i} \frac{A_{i}}{\nu_{i}^{2}} \frac{d \ln \nu_{i}}{d \ln \ell}\right]
$$




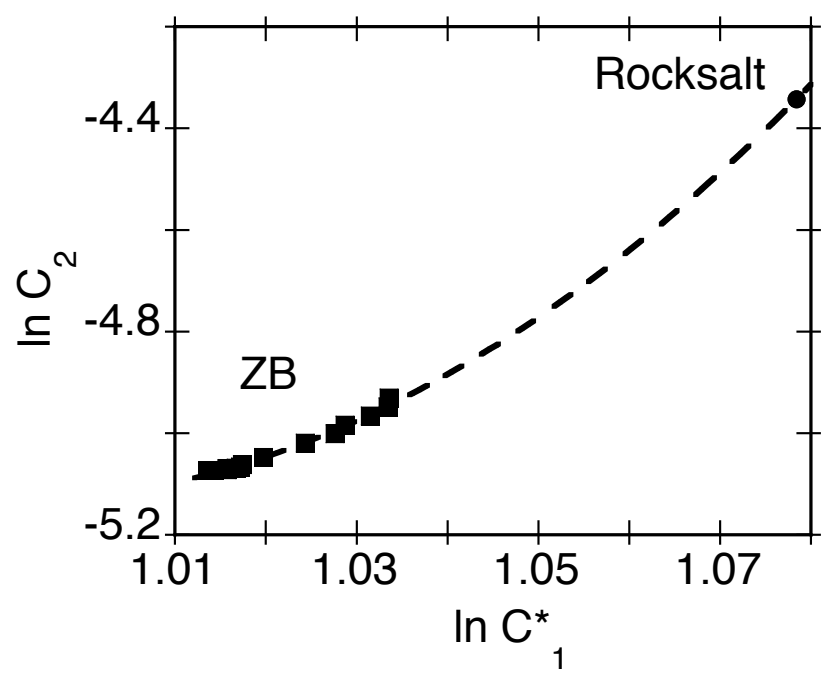

Figure 16. Logarithm of the second cumulant plotted as a function of the logarithm of the first cumulant in the ZB and RS phases (squares and circle, respectively).

The resulting linear GP is thus, within the QHA,

$$
\gamma_{\text {lin }}=\frac{\sum_{i}\left(A_{i} / \nu_{i}^{2}\right) \gamma_{i}^{\text {lin }}}{\sum_{i} A_{i} / \nu_{i}^{2}}
$$

For CdTe, the thermodynamic GP evaluated at $300 \mathrm{~K}$ from the experimental coefficient of lattice thermal expansion, specific heat and compressibility is $\gamma_{v}=0.6$ $[77,78]$; the same value has been recently obtained by ab-initio lattice dynamics calculations [79]; this value would correspond to a linear GP three times larger, $\gamma_{\text {lin }}=1.8$, much smaller than the bond GP $\gamma_{\mathrm{b}, T} \simeq 6$ obtained in the present work at $300 \mathrm{~K}$.

According to calculations from first principles [68], the mode GPs of CdTe are negative for the TA modes in a large part of the first Brillouin Zone (values between 0 and -2.5). The GPs of the other modes are included between 0 and 2.5. Even taking into account that the linear GPs are three times larger than the volume GPs, it is not reasonable to expect that the weighted sum (20) can be as high as the experimental bond $\mathrm{GP} \gamma_{\mathrm{b}, T} \simeq 6$ at $300 \mathrm{~K}$ and $100 \mathrm{kPa}$.

The connection between the bond GP and the mode GPs expressed by (19) is thus not verified in the present case of CdTe. This result is consistent with the hypothesis that the QHA is insufficient to account for the local vibrational properties of CdTe. Actually, the effective pair potential connected to the nearest-neighbour distribution sampled by EXAFS maintains a relatively strong memory of the anharmonicity of the single pair potential [80], which is instead less influent on the distributions of outer-shell distances and is expected to be weak in the de-localized normal modes.

5.3.4. Relation between first and second cumulant Let us now try to gain a deeper understanding of the reduction of the second cumulant when the pressure increases in 
the ZB phase and of its relatively higher value in the RS phase.

In the classical approximation (4), the second cumulant is to first order proportional to the temperature and inversely proportional to the square of the Einstein frequency; taking into account the relation between bond GP, bond distance and frequency, one easily derives the differential relation

$$
\frac{d C_{2}}{C_{2}}=2 \gamma_{b} \frac{d C_{1}^{*}}{C_{1}^{*}}+\frac{d T}{T} .
$$

For constant- $T$ pressure-dependent measurements, the last term $d T / T$ is zero and $\gamma_{b}=\gamma_{b, T}$.

The relation between the logarithms of the first and second cumulant of CdTe at constant temperature when the pressure is varied is shown in figure 16; both the ZB and RS phases are here considered. In the ZB phase (squares), the relation is not linear, consistent with the reduction of the bond GP from 6 to 2 when the pressure increases. In the RS phase (circle), the values of both first and second cumulant are larger than in the ZB phase; it is significant that the values for the RS phase are consistent with the extrapolation to high pressures of the behaviour of the ZB phase (dashed line). This result suggests that the large Debye-Waller factor in the RS phase is of purely vibrational origin, and according to (21) depends on the increase of the bond distance via the GP, say it is an effect of anharmonicity. No significant structural contributions are thus necessary to explain the large Debye-Waller factor in the RS phase.

An approach similar to that of (21), although in finite form, has been used by Willis and Pryor to discuss the anharmonicity effects on the atomic temperature factors of diffraction [58] and extended by Tranquada and Ingalls to the pressure-dependent EXAFS measurements on $\mathrm{CuBr}$ [16]. Notice that in both cases the volume GP obtained from the QHA treatment of thermal expansion was considered.

\section{Conclusions}

EXAFS and diffraction measurements have been made on CdTe at room temperature in the pressure range from $100 \mathrm{kPa}$ to $5 \mathrm{GPa}$. The three phases (ZB, mixed ZB-cinnabar, $\mathrm{RS}$ ) are well singled out in the diffraction patterns and in the Fourier Transforms of the EXAFS functions. The quantitative analysis of EXAFS has been mainly focussed on the low-pressure ZB phase.

The bond compressibility in the ZB phase has been found to decrease faster than the lattice compressibility when the pressure increases; this behaviour has been attributed to the effect of vibrations perpendicular to the bond direction, which allow the bond length to be less stressed than the lattice parameter.

The increase of pressure induces a slight strenghtening of the effective pair potential and a large shift of its position towards smaller distances. The reduction of the parallel MSRD is accompanied by an increase of the perpendicular MSRD, so that the ellipsoid of relative vibrations becomes more anisotropic when the pressure is increased. This 
behaviour has been attributed to the increasing weight of normal modes with negative GP when the pressure increases.

From the variations of Einstein frequency and bond distance, a bond GP can be defined and measured. One has found that the constant- $T$ bond GP is smaller than the constant- $p$ bond GP. The difference is mainly due to the different variation of the bond distance. The relation of the constant-temperature bond GPs with the thermodynamic GP and with the mode GPs has been explored. One finds that the values of bond GP measured by EXAFS are larger than the values calculated from the mode GPs. This result suggests the inadequacy of the QHA to deal with the local vibrational properties measured by EXAFS.

In the RS phase, the second cumulant is much larger than in the ZB phase. The large value of the second cumulant can be justified on purely vibrational terms as an anharmonicity effect connected to the increase of the bond distance. No assumption of the presence of structural disorder is necessary.

The results presented here for CdTe confirm once more the difference between local and average properties of crystals and suggest that these differences can be best evidenced by joint EXAFS and diffraction measurements performed as a function not only of temperature but also of pressure. The knowledge of local properties is complementary to the one of average properties and allows a deeper understanding of the global behaviour of materials. Some local properties are more easily measured than the corresponding average properties: it is the case, for example, of the parallel and perpendicular MSRDs with respect to the atomic displacement parameters or of the bond GPs with respect to the mode GPs. The bond GPs can be interesting for comparing the local properties of different materials and for validating theoretical calculations.

\section{Acknowledgments}

We acknowledge the European Synchrotron Radiation Facility (ESRF) for provision of synchrotron radiation facilities. Virginia Monteseguro Padron and Raffaela Torchio helped in preparing and loading the sample in the diamond anvil cell. The research has been supported by PRIUS (Premier Research Institute for Ultrahigh-pressure Sciences), project FY2016.

\section{References}

[1] Mikkelsen, Jr J C and Boyce J B 1983 Phys. Rev. B 28 7130-7140

[2] Beni G and Platzman P M 1976 Phys. Rev. B 14 1514-1518

[3] Busing W R and Levy H A 1964 Acta Crystallogr. 17 142-146

[4] Dalba G, Fornasini P, Gotter R and Rocca F 1995 Phys. Rev. B 52 149-157

[5] Stern E A 1997 J. Phys. IV France 7 137-140

[6] Dalba G, Fornasini P, Grisenti R and Purans J 1999 Phys. Rev. Lett. 82 4240-4243

[7] Fornasini P and Grisenti R 2014 J. Chem. Phys. 141164503 
[8] Fornasini P 2015 Vibrational anisotropy. X-ray Absorption Spectroscopy of Semiconductors ed Schnohr C S and Ridgway M C (New York: Springer) chap 6, pp 127-141

[9] Fornasini P, Grisenti R, Dapiaggi M, Agostini G and Miyanaga T 2017 J. Chem. Phys. 147044503

[10] Fornasini P and Grisenti R 2015 J. Synchrotron Rad. 22 1242-1257

[11] Barron T H K 1998 Generalized theory of thermal expansion of solids CINDAS data series on material properties, Vol. I-4, Thermal expansion of solids ed Ho C Y

[12] Aschroft N W and Mermin N D 1976 Solid State Physics (Thomson Brooks/Cole)

[13] Li C W, Tang X, Muñoz J A, Keith J B, Tracy S J, Abernathy D L and Fultz B 2011 Phys. Rev. Lett. 107195504

[14] Piskunov S, Žguns P A, Bocharov D, Kuzmin A, Purans J, Kalinko A, Evarestov R A, Ali S E and Rocca F 2016 Phys. Rev. B 93214101

[15] Ingalls R, Garcia G A and Stern E A 1978 Phys. Rev. Lett. 40 334-336

[16] Tranquada J M and Ingalls R 1986 Phys. Rev. B 34 4267-4277

[17] Freund J, Ingalls R and Crozier E D 1989 Phys. Rev. B 39 12537-12547

[18] San-Miguel A, Polian A, Gauthier M and Itié J P 1993 Phys. Rev. B 48 8683-8693

[19] Di Cicco A, Filipponi A, Itié J P and Polian A 1996 Phys. Rev. B 54 9086-9098

[20] Itié J P, Baudelet F and Rueff J P 2016 High pressure xas, xmcd and ixs. X-Ray absorption and X-Ray Emission Spectroscopy. ed van Bokhoven J A and Lamberti C (Wiley) chap 14, pp $385-405$

[21] Pellicer-Porres J, Segura A, Ferrer Ch, Muñoz V, San Miguel A, Polian A, Itié J P, Gauthier M and Pascarelli S 2002 Phys. Rev. B 65174103

[22] Poloni R, Fernandez-Serra M V, Le Floch S, De Panfilis S, Toulemonde P, Machon D, Crichton W, Pascarelli S and San-Miguel A 2008 Phys. Rev. B 77035429

[23] Pellicer-Porres J, Segura A, Muñoz A, Polian A and Congeduti A 2010 J. Phys.: Condens. Matter 22045701

[24] Fabbris G, Hücker M, Gu G D, Tranquada J M and Haskel D 2013 Phys. Rev. B 88060507

[25] Itié J P, Polian A, Baudelet F, Mocuta C, Thiaudière D, Fonda E and Irifune T 2016 High Pressure Research 36 479-492

[26] Hung N V, Hung V V, Hieu H K and Frahm R R 2011 Physica B 406 456-460

[27] Hieu H K and Hung V V 2013 High Press. Res. 33 768-776

[28] Duc N B, Tho V Q, Tien T S, Khoa D Q and Hieu H K 2018 Radiat. Phys. Chem. 149 61-64

[29] Irifune T, Kurio A, Sakamoto S, Inoue T and Sumiya H 2003 Nature (London) 421599

[30] Ishimatsu N, Matsumoto K, Maruyama H, Kawamura N, Mizumaki M, Sumiya H and Irifune T 2012 J. Synchrotron Rad. 19768

[31] Ishimatsu N, Kawamura N, Mizumaki M, Maruyama H, Sumiya H and Irifune T 2016 High Pressure Research 36381

[32] Baldini M, Yang W, Aquilanti G, Zhang L, Ding Y, Pascarelli S and Mao W L 2011 Phys. Rev. B 84014111

[33] Dewaele A, Worth N, Pickard C J, Needs R J, Pascarelli S, Mathon O, Mezouar M and Irifune T 2014 Nature Chemistry 8 784-790

[34] Sanson A, Kantor I, Cerantola V, Irifune T, Carnera A and Pascarelli S 2016 Phys. Rev. B 94 014112

[35] Mathon O, Beteva A, Borrel J, Bugnazet D, Gatla S, Hino R, Kantor I, Mairs T, Munoz M, Pasternak S, Perrina F and Pascarelli S 2015 J. Synchrotron Rad. 22 1548-1554

[36] Ziegler E, Aquilanti G, Mathon O, de Panfilis S and van Vaerenbergh P 2009 X-ray Spectrom. 38 250

[37] De Panfilis S, Gorelli F, Santoro M, Ulivi L, Gregoryanz E, Irifune T, Shinmei T, Kantor I, Mathon O and Pascarelli S 2015 J. Chem. Phys. 142214503

[38] Abd el All N, Dalba G, Diop D, Fornasini P, Grisenti R, Mathon O, Rocca F, Thiodjio Sendja B and Vaccari M 2012 J. Phys.: Condens. Matter 24115403

[39] Abd el All N, Thiodjio Sendja B, Grisenti R, Rocca F, Diop D, Mathon O, Pascarelli S and 
Fornasini P 2013 J. Synchrotron Rad. 20 603-613

[40] Mujica A, Rubio A, Muñoz A and Needs R J 2003 Rev. Mod. Phys. 75 863-912

[41] Nelmes R J, McMahon M I, Wright N G and Allan D R 1993 Phys. Rev. B 48 1314-1317

[42] McMahon M I, Nelmes R J, Wright N G and Allan D R 1993 Phys. Rev. B 48 16246-16251

[43] Nelmes R J, McMahon M I, Wright N G and Allan D R 1995 Phys. Rev. B 51 15723-15731

[44] Côté M, Zakharov O, Rubio A and Cohen M L 1997 Phys. Rev. B 55 13025-13031

[45] Letoullec R, Pinceaux J P and Loubeyre P 1988 High Pressure Research 1 77-90

[46] Klotz S, Chervin J C, Munsch P and Le Marchand G 2009 J. Phys. D: Appl. Phys. 42075413

[47] Cliffe M J, Dove M T, Drabold D A and Goodwin A L 2010 Phys. Rev. Lett. 104125501

[48] Vaccari M, Grisenti R, Fornasini P, Rocca F and Sanson A 2007 Phys. Rev. B 75184307

[49] Bunker G 1983 Nucl. Instrum. Methods Phys. Res. 207 437-444

[50] Tranquada J M and Ingalls R 1983 Phys. Rev. B 28 3520-3528

[51] Dalba G, Fornasini P and Rocca F 1993 Phys. Rev. B 47 8502-8514

[52] Rehr J J, Albers R C and Zabinsky S I 1992 Phys. Rev. Lett. 693397

[53] Ankudinov A L, Ravel B, Rehr J J and Conradson S D 1998 Phys. Rev. B 587565

[54] Newville M 2001 J. Synchrotron Rad. 8 314-316

[55] Ravel B and Newville M 2005 J. Synchrotron Rad. 12 537-541

[56] Hammersley A P, Svensson S O, Hanfland M, Fitch A N and Hausermann D 1996 High Pressure Research 14 235-248

[57] Lutterotti L, Matthies S, Wenk H R, Schultz A S and Jr J W R 1997 Journal of Applied Physics 81 594-600

[58] Willis B T M and Pryor A W 1975 Thermal Vibrations in Crystallography (Cambridge University Press)

[59] Ahmed S I, Aquilanti G, Novello N, Olivi L, Grisenti R and Fornasini P 2013 J. Chem. Phys. 139 164512

[60] Murnaghan F D 1944 Proc. Nat. Acad. Sci. 30 244-247

[61] Frenkel A I and Rehr J J 1993 Phys. Rev. B 48 585-588

[62] Yokoyama T 1999 J. Synchrotron Rad. 6 323-325

[63] Stern E A, Livins P and Zhang Z 1991 Phys. Rev. B 43 8850-8860

[64] Martínez-Garcia D, Le Godec Y, Mézouar M, Syfosse G, Itié J P and Besson J M 1999 Phys. Stat. Sol. (b) 211 461-467

[65] Cramér H 1966 Mathematical Methods of Statistics (Princeton University Press)

[66] Horning R D and Staudenmann J L 1986 Phys. Rev. B 34 3970-3979

[67] Vaccari M and Fornasini P 2006 J. Synchrotron Rad. 13 321-325

[68] Matsuo Kagaya H and Soma T 1986 Phys. Stat. Sol. (b) 138 K13-K16

[69] Basak T, Rao M N, Gupta M K and Chaplot S L 2012 J. Phys.: Condens. Matter 24115401

[70] Soma T and Matsuo Kagaya H 1986 Phys. Stat. Sol. (b) 135 K103-K106

[71] Maier B J, Angel R J, Mihailova B, Marshall W G, Gospodinov M and Bismayer U 2011 J. Phys.: Condens. Matter 23035902

[72] Brüesch P 1982 Phonons: theory and experiment. I. Lattice Dynamics and Models of Interatomic Forces (Springer Series in Solid State Sciences vol 34) (Springer, Berlin)

[73] Boehler R 1983 Phys. Rev. B 276754

[74] Boehler R and Ross M 1984 Phys. Rev. B 293673

[75] Pandya C V, Vyas P R, Pandya T C and Gohel V B 2002 Bull. Mater. Sci. 25 63-67

[76] Fang Z H 1996 J. Phys.: Condens. Matter 8 7067-7071

[77] Novikova S I 1961 Soviet Phys. - Solid State 2 2087-2089

[78] Smith T F and White G K 1975 J. Phys. C: Solid State Phys. 8 2031-2042

[79] Wang L, Yuan P F, Wang F, Sun Q, Guo Z X, Liang E J and Jia Y 2014 Mater. Chem. Phys. $148214-222$

[80] Hung V N and Fornasini P 2007 J. Phys. Soc. Jpn 76084601 